\title{
FC-98 Regulates TLR9-Mediated of CXCL-10 Expression in Dendritic Cells via MAPK and STAT1 Signaling Pathway
}

\author{
Yonghong Yang, ${ }^{1}$ Huan Dou, ${ }^{1,2}$ Xiaoqin Li, ${ }^{1}$ Yuxian Song, \\ Wei Gong, ${ }^{1}$ Renxiang Tan, ${ }^{3}$ and Yayi Hou ${ }^{1,2}$ \\ ${ }^{1}$ The State Key Laboratory of Pharmaceutical Biotechnology, Division of Immunology, Medical School, Nanjing University, \\ Nanjing 210093, China \\ 2 Jiangsu Key Laboratory of Molecular Medicine, Nanjing 210093, China \\ ${ }^{3}$ Institute of Functional Biomolecules, School of Life Sciences, Nanjing University, Nanjing 210093, China
}

Correspondence should be addressed to Huan Dou; douhuan@nju.edu.cn and Yayi Hou; yayihou@nju.edu.cn

Received 22 October 2013; Revised 17 December 2013; Accepted 31 December 2013; Published 17 February 2014

Academic Editor: Thomas Griffith

Copyright (C) 2014 Yonghong Yang et al. This is an open access article distributed under the Creative Commons Attribution License, which permits unrestricted use, distribution, and reproduction in any medium, provided the original work is properly cited.

\begin{abstract}
Dendritic cells (DCs), as the most potent professional antigen presenting cells, play a crucial role in both innate and adaptive immune systems. Genomic bacterial DNA mimicked by unmethylated CpG motifs is discovered to possess immunostimulatory effects. CpG-DNA recognized by Toll-like receptor 9 (TLR9) on DCs arouses many immune diseases (such as cancer, viral infection, and autoimmune disorders). In this study we investigated the effects of FC-98 on CpG-induced bone marrow-derived DCs (BMDCs). The results showed that FC-98 significantly inhibited the CpG-induced BMDCs maturation and function by suppressing the expression of surface markers (CD40, CD80, CD86, and MHCII). Moreover, FC-98 downregulated the expression of C-X-C motif chemokine 10 (CXCL-10) both at the mRNA and protein level after CpG induction. Meanwhile, FC-98 markedly affected the migration of BMDCs to T cells without affecting their endocytosis capacity. Furthermore, FC-98 was confirmed to decrease CXCL-10 expression by inhibiting CpG-induced activation of MAPKs (ERK, JNK, and p38) and STAT1 signaling. Overall, these results suggested that FC-98 was a potential molecule in the treatment of CXCL-10-mediated immune diseases.
\end{abstract}

\section{Introduction}

Dendritic cells (DCs) are the most potent professional antigen presenting cells and play a crucial role in both innate and adaptive immune systems [1]. Indeed, there is compelling evidence that DCs participate in the induction and treatment of numerous diseases, such as autoimmune diseases [2, 3], cancer [4], and infection [5]. DCs are derived from bone marrow progenitor cells and exist in vivo at two different stages: immature and nature. In the steady state, most DCs are immature [6]. When exposed to stimuli, DCs capture antigens and migrate to draining lymph nodes, where they present antigens to $\mathrm{T}$ lymphocytes. This process involves endocytosis, the upregulation of cell-surface molecules markers (CD40, CD80, CD86, and MHCII) [7], and the large quantity synthesis of chemokines and cytokines [8]. Moreover, DCs can recognize and response to pathogen-associated molecular patterns through pattern recognition receptors including Toll-like receptors (TLRs) family [9]. To date, 10 TLRs in human and 13 TLRs in mouse have been identified, respectively [10]. Binding of ligands to TLRs results in maturation of DCs and initiation of the adaptive immune responses [11]. Recently genomic bacterial DNA mimicked by unmethylated $\mathrm{CpG}$ motifs is discovered to possess immunostimulatory effects [12]. CpGDNA receptor, Toll-like receptor 9 (TLR9), is also expressed by DCs. TLR9 locates in endosomes and is responsible for sensing unmethylated CpG-DNA, discriminates between self- and foreign DNA, and plays an important role in many immune diseases such as autoimmune diseases and cancer [13]. Synthetic oligodeoxynucleotides (CpG-ODN) have been found taking part in promoting activation and maturation of bone marrow-derived dendritic cells (BMDCs) [14].

It was reported that the expression of $\mathrm{C}-\mathrm{X}-\mathrm{C}$ motif chemokine 10 (CXCL-10) by DCs is elevated following CpGODN stimulation $[15,16]$. CXCL-10, also named as interferon 
gamma-induced protein 10 (IP-10), is a member of CXC chemokines and known as activator on antitumor, antiviral, antifungal, and autoimmune diseases [17-19]. CXCL-10 also plays an important role in the interaction between DCs and $\mathrm{T}$ cells through binding and activating its receptor CXCR3, expressing on the surface of T cells [20]. Thus the interaction influences the recruitment and activation of Th1polarized cells [21]. Recently, more and more attention was paid to the potential of CXCL-10 signaling pathway. In human macrophages, microglia, and epithelial and cancer cells, CXCL-10 expression has been demonstrated to be mediated through MAPK (ERK1/2, JNK, and p38) and STAT1 signaling pathway [22-24]. However, the regulation pathway of CXCL10 in BMDCs is still not clear.

Our previous study demonstrated that FC-98, a benzenediamine derivate, inhibited the TNF- $\alpha$, IL-6, MCP-1, and NO production from LPS-induced murine macrophages through attenuating the activation of NF- $\kappa \mathrm{B}$, JNK, and IRF3 signaling pathway. Moreover, FC-98 effectively improved the survival rate and injured organ of mice's sepsis model (unpublished). However, it is still unclear whether FC-98 has effects on DCs and the underling mechanisms. In this study, we attempted to investigate the effects of FC-98 on the maturation of $\mathrm{CpG}$-induced BMDCs and their functions. We showed that FC-98 markedly inhibited the expression of costimulatory molecules of BMDCs without affecting their endocytosis capacity. Moreover, FC-98 significantly inhibited the expression of CXCL-10 both on mRNA and protein level, leading to decreasing migration ability of T cells to DCs. As expected, FC-98 blocked both MAPK and STAT1 signaling pathway upstream the CXCL-10. For the first time FC-98 was approved to be a potent compound for treatment of TLR9-mediated and CXCL-10-assosiated autoimmune and inflammatory diseases.

\section{Materials and Methods}

2.1. Reagents, Antibodies, and Mice. FC-98 ( ${ }^{1}-[(4-$ fluorophenyl)methyl]-4-methyl-1,2-benzenediamine, purity $>99 \%$, chemical structure shown in Figure 1) was commercially synthesized as previously reported [25]. For testing, FC-98 was dissolved in dimethyl sulfoxide (DMSO) at a concentration of $50 \mathrm{mM}$ as a stock solution and diluted to the desired concentration with medium before each experiment. RPMI 1640, fetal bovine serum (FBS), penicillin, and streptomycin were purchased from Gibco Inc. (Grand Island, NY, USA). Recombinant granulocyte-macrophage colony-stimulating factor (GM-CSF) was from Miltenyi Biotec (Bergisch Gladbach, Germany), and recombinant interleukin-4 (IL-4) was from PeproTech, Inc. (Rocky Hill, NJ). CpG-ODN 1668 was synthesized by Invitrogen (Shanghai, China). FITC Annexin V Apoptosis Detection Kit was purchased from $\mathrm{BD}$ Pharmingen (Heidelberg, Germany). Cell counting kit-8 (CCK-8) was purchased from DojinDo Laboratories (Kyushu, Japan). Mouse CXCL-10 enzyme-linked immunosorbent assay (ELISA) kit was purchased from R\&D Systems (Minneapolis, MN, USA). Antibodies (Abs) used to detect the expression of CD11c,

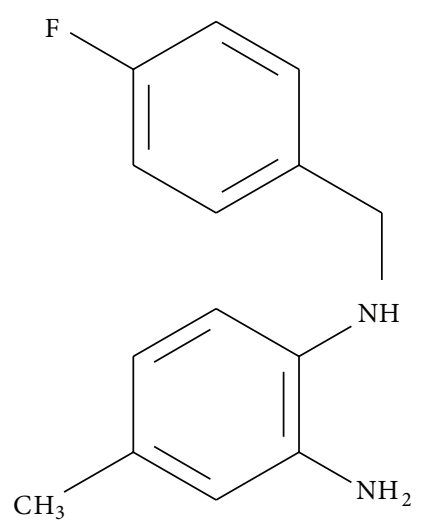

FIGURE 1: Stereostructure of structure of FC-98.

CD40, CD80, CD86, and MHCII as well as isotype-matched control mAbs were purchased from eBioscience (San Diego, CA, USA). FITC-dextran was from Sigma-Aldrich (St. Louis, MO, USA). BCA kit was purchased from Pierce (Rockford, IL). Anti-mouse JNK, phospho-JNK, p38, phospho-p38, ERK, phospho-ERK, STAT1, phospho-STAT1, Tublin, and GAPDH for Western blot analysis were purchased from Cell Signaling Technology (Danvers, MA, USA). Female C57BL/6 mice 4-6 weeks old were purchased from the Animal Research Center of Yangzhou University (Yangzhou, China). All of the animals were maintained under specific pathogen-free conditions and acclimatized for at least 1 week to the surrounding environment prior to use. Experiments were conducted according to institutional animal ethics guidelines.

\subsection{Generation of Mouse Bone Marrow Derived Dendritic} Cells. Bone marrow cells were obtained from the tibiae and femurs of C57BL/6 mice and treated with red blood cell lysing buffer. Cells were cultured in RPMI-1640 medium supplemented with $10 \% \mathrm{FBS}, 100 \mu \mathrm{g} / \mathrm{mL}$ penicillin, $100 \mu \mathrm{g} / \mathrm{mL}$ streptomycin, $10 \mathrm{ng} / \mathrm{mL}$ murine GM-CSF, and $1 \mathrm{ng} / \mathrm{mL}$ IL-4 at a density of $1 \times 10^{6}$ cells $/ \mathrm{mL}$. The plates were incubated at $37^{\circ} \mathrm{C}$ in $5 \% \mathrm{CO}_{2}$ for 7 days and half of the medium was replaced by fresh medium containing GM-CSF and IL-4 on days 3 and 5 . At day 7, nonadherent and loosely adherent cells were harvested and transferred into new culture plates for further experiments.

2.3. Cell Viability Assay. The cytotoxicity of FC-98 was evaluated using the Cell Counting Kit 8 (CCK-8) according to the manufacturer's instructions. In brief, $100 \mu \mathrm{L}$ of $5 \times 10^{3}$ DCs was seeded in 96-well plates and incubated overnight and cells were then incubated with various concentrations of FC-98 for $24 \mathrm{~h}$. Subsequently, $10 \mu \mathrm{L}$ of the CCK- 8 solution was added to each well and incubated for another $3 \mathrm{~h}$. The absorbance at $450 \mathrm{~nm}$ was measured using microplate reader (Synergy HT, Bio-Tek).

2.4. Assessment of Apoptosis of DCs. The extent of apoptosis in DCs was assayed by the Annexin V/PI staining. In brief, 
DCs were cultured with various concentrations of FC-98 for $24 \mathrm{~h}$ at $37^{\circ} \mathrm{C}$. After washing twice with cold medium phosphate buffered saline (PBS), cells were incubated with Annexin V-FITC in dark for $5 \mathrm{~min}$, and then PI was added before analysis of samples with flow cytometry. Flow cytometry was performed by using FACS Calibur system (BD Biosciences, Franklin Lakes, NJ, USA). And the data were analyzed by WinMDI (Scripps Institute, La Jolla, CA).

2.5. Flow Cytometric Analysis of Surface Markers. After treatment, DCs were washed twice in FACS medium containing $1 \%$ FCS and $0.1 \% \mathrm{NaN}_{3}$. Subsequently, cells were incubated for 30 minutes at $4^{\circ} \mathrm{C}$ with appropriately diluted antibodies of CD80, CD86, CD40, and MHCII according to the standard procedure. Cells were washed and fixed in $4 \%$ paraformaldehyde. An isotype control was used for each antibody. Fluorescence was measured using flow cytometry detection, and data were analyzed using Cell Quest software (BD Biosciences, Franklin Lakes, NJ, USA). The results of all experiments were expressed as mean fluorescent intensity (MFI).

2.6. Endocytosis Assay. Assay of DCs endocytotic activity was measured by uptake of FITC-dextran and quantitated using flow cytometry. In brief, $2 \times 10^{5}$ DCs were incubated with various concentrations of FC-98 for $2 \mathrm{~h}$, and then FITC-dextran (final concentration $0.5 \mathrm{mg} / \mathrm{mL}$ ) was added into the culture medium at $37^{\circ} \mathrm{C}$ in dark for $1 \mathrm{~h}$. Then cells were collected and washed 3 times with cold PBS to stop endocytosis. Trypan blue $(1.2 \mathrm{mg} / \mathrm{mL})$ was used to quench the endocytic activity and remove any free FITC-dextran. Cells were analyzed by flow cytometric analysis. A parallel experiment performed at $4^{\circ} \mathrm{C}$ served as a negative control.

\subsection{RNA Isolation, Reverse Transcription Polymerase Chain} Reaction, and Q-PCR. Total RNA was extracted from $2 \times$ $10^{6}$ cells using TRIZOL (Invitrogen, USA) following the manufacturer's description. The RNA quantity was determined by spectrophotometer at $260 \mathrm{~nm}$. $20 \mu \mathrm{L}$ cDNA was synthesized from $1 \mu \mathrm{g}$ total RNA using a revert aid firststrand cDNA synthesis kit (Fermentas). Quantitative realtime PCR analysis was performed in $20 \mu \mathrm{L}$ PCR reaction mixture using the 7300 PCR System (Life Technologies, Grand Island, NY, USA). Amplification conditions were $95^{\circ} \mathrm{C}$ for $10 \mathrm{~min}$, and 40 cycles of $95^{\circ} \mathrm{C}$ for $30 \mathrm{~s}, 60^{\circ} \mathrm{C}$ for $30 \mathrm{~s}$, and $72^{\circ} \mathrm{C}$ for $30 \mathrm{~s}$. The mRNA levels for target gene were normalized to $\beta$-actin in the same sample. The primer sequences used in this study were as follows: CXCL10, $5^{\prime}$-CCAAGTGCTGCCGTCATTTTC-3' (forward) and $5^{\prime}$-GGCTCGCAGGGATGATTTCAA- $3^{\prime}$ (reverse); GAPDH, $5^{\prime}$-GGTGAAGGTCGGTGTGAACG-3' (forward) and $5^{\prime}$ CTCGCTCCTGGAAGATGGTG-3' (reverse).

2.8. ELISA Assay. BMDCs were treated as experiment designing. Supernatants were harvested after $24 \mathrm{~h}$ culture and assayed for CXCL-10 expression according to manufacturer's instructions. In brief, cell culture supernatants and CXCL-10 standards in triplicate were added to precoated 96-well plate and incubated for $2 \mathrm{~h}$ at $22-25^{\circ} \mathrm{C}$. Afterward, the plate was washed 5 times with washing buffer. Then, HRP-conjugated secondary antibody was added and the plate was incubated for $2 \mathrm{~h}$ at $22-25^{\circ} \mathrm{C}$. After washing 5 times, substrate solution were added and incubated for $30 \mathrm{~min}$ at $22-25^{\circ} \mathrm{C}$ in the dark. The stop solution was then added to each well and the absorbance was read at $450 \mathrm{~nm}$ using a microplate reader.

2.9. Chemotaxis Assay. To assess the effect of FC-98 on $\mathrm{T}$ cells transmigration to BMDCs, Chemotaxis assay were performed using a 24 well Transwell system with $5 \mu \mathrm{m}$ pore polycarbonate filter (Corning, NY, USA). T cells were purified from lymph node, and $1 \times 10^{6} \mathrm{~T}$ cells in $100 \mu \mathrm{L}$ of RPMI 1640 were added to the upper chamber and $600 \mu \mathrm{L}$ supernatant of DCs was added to the lower chamber. After $3 \mathrm{~h}$ incubation at $37^{\circ} \mathrm{C}$, cells that had migrated into lower wells were harvested and counted by flow cytometry.

2.10. Western Blot Analysis. About $1 \times 10^{6}$ DCs were lysed in ice-cold buffer ( $0 \mathrm{mM}$ Tris- $\mathrm{HCl}(\mathrm{pH}$ 7.6), $250 \mathrm{mM} \mathrm{NaCl}$, $0.5 \% \mathrm{NP}-40,3 \mathrm{mM}$ EDTA, and $1.5 \mathrm{mM}$ EGTA) containing $10 \mu \mathrm{g} / \mathrm{mL}$ aprotinin, $10 \mu \mathrm{g} / \mathrm{mL}$ leupeptin, $1 \mathrm{mM}$ DTT, $1 \mathrm{mM}$ PNPP, and $0.1 \mathrm{mM} \mathrm{Na} \mathrm{NO}_{4}$ for $30 \mathrm{~min}$. After centrifugation, cell lysates were collected and measured by the BCA Protein Assay Reagent Kit (Pierce, Biotechnology). Equal amounts of protein from each sample were mixed with 1X loading buffer and denatured at $95^{\circ} \mathrm{C}$ for $5 \mathrm{~min}$. Then they were subjected to $10 \%$ SDS-PAGE and transferred on to polyvinylidene difluoride (PVDF) membranes (Roche, Germany). After the PVDF membranes were blocked for $2 \mathrm{~h}$ with $5 \%$ bovine serum albumin in $0.1 \%$ Tween-20/TBS, the membranes were incubated overnight with primary antibodies at a concentration of $1: 1000$ at $4^{\circ} \mathrm{C}$. After washing, the membranes were incubated with HRP-conjugated secondary antibody at 1:3000 dilutions for $2 \mathrm{~h}$ at the room temperature and then washed again. Protein bands were detected by enhanced chemiluminescence Western blotting detection reagents using ECL method, and images were acquired by FluorChem FC2 System (Alpha Innotech Corporation, USA). The membrane was stripped and reprobed with GAPDH or Tublin antibody to equal loading of proteins.

2.11. Assay of Luciferase Reporter Gene Expression. BMDCs were seeded in 24-well plates at $1 \times 10^{5}$ cells per well and incubated overnight. Cells were cotransfected with $100 \mathrm{ng}$ pAP-1 luciferase reporter plasmids and $10 \mathrm{ng}$ pRL-TK-Renilla luciferase plasmids using Lipofectamine LTX reagent. Total amounts of plasmid DNA were equalized with pGL6 (empty control vector). After $24 \mathrm{~h}$ of culture, cells were pretreated with FC-98 for $1 \mathrm{~h}$ and then stimulated with $1 \mu \mathrm{M}$ CpG for another $6 \mathrm{~h}$. Luciferase activities were measured using the Dual-Luciferase Reporter Assay system (Promega, Madison, WI) according to the manufacturer's instructions. Data are normalized for transfection efficiency by dividing Firefly luciferase activity with that of Renilla luciferase.

2.12. Statistical Analysis. All results were analyzed with Prism 5.0 (GraphPad Software, Inc., San Diego, CA) and expressed 
as means \pm SD Data analysis was done using Student's $t$-test and one-way analysis of variance (ANOVA). Differences with $P<0.05$ were considered significant.

\section{Results}

3.1. FC-98 Had No Influence on BMDCs Viability and Apoptosis. FC-98 has a molecular weight 290 and its stereostructure is shown in Figure 1. In the initial series of experiments, we evaluated the effect of FC-98 on the cell viability of BMDCs by CCK- 8 assay. BMDCs were isolated and purified as described in Section 2 and then exposed to various concentrations ( 0 , $25,100,200$, and $400 \mu \mathrm{M}$ ) of FC-98 for $24 \mathrm{~h}$. The viability of the cells was determined at the wavelength of $450 \mathrm{~nm}$. The results showed that FC-98 did not affect the cell viability up to $200 \mu \mathrm{M}$ (Figure $2(\mathrm{a})$ ).

To further explore the cytotoxicity of FC-98 on DCs, we performed the Annexin V/PI costaining flow cytometry analysis. BMDCs were harvested and cultured in 24-well plate, and then a range of $0-250 \mu \mathrm{M}$ FC-98 were added. After $24 \mathrm{~h}$ coculture, the cells were washed and stained for Annexin $\mathrm{V}$ and PI. Flow cytometry analysis was used to determine the cell viability after FC-98 treatment on DCs. No significant difference in Annexin $\mathrm{V}^{-} / \mathrm{PI}^{-}$was detected between BMDCs treated with FC-98 at the concentrations from 0 to $200 \mu \mathrm{M}$ (Figure 2(b)). Taken together, the concentration of FC-98 $\leq 200 \mu \mathrm{M}$ was selected for the following experiments.

3.2. FC-98 Suppressed the Phenotypic Maturation of CpGInduced DCs without Influence on Endocytosis. When DCs detect exogenous danger such as bacterial DNA, the maturation will be initiated immediately. The matured DCs express high levels of costimulatory molecules (CD40, CD80, and CD86) and MHCII. To determine the effects of FC-98 on the maturation of DCs, immature DCs were exposed to different concentrations of FC-98 for $2 \mathrm{~h}$, followed by $24 \mathrm{~h}$ stimulation of CpG $(1 \mu \mathrm{M})$. The expression of MHCII, CD40, CD80, and CD86 were assayed by flow cytometry. As shown in Figure 3(a), FC-98 alone had little effect on the MFI of MHCII and costimulatory molecules on BMDCs compared with control group. In contrast, these molecules expressions on BMDCs pretreatment with FC-98 were sufficiently reduced, since the expression of surface markers was significantly upregulated with treatment of $\mathrm{CpG}$.

DCs are the most potent professional antigen capturing and presenting cells. Endocytosis is thought to be characteristic during the process of pathogen uptake. To examine the endocytosis of BMDCs, we treated BMDCs as indicated and then used $0.5 \mathrm{mg} / \mathrm{mL}$ FITC-labeled Dextran to quantify the endocytic capacity by flow cytometry. Figure 3(b), the similar endocytic capacity was observed in BMDCs both in $37^{\circ} \mathrm{C}$ and $4^{\circ} \mathrm{C}$ after FC-98 treatment. This indicated that FC-98 had no influence on the endocytic function of BMDCs.

3.3. FC-98 Suppressed the Expression and Function of CXCL10 in CPG-Induced BMDCs. CXCL-10 plays an important role in the interaction between DCs with T cells. To further determine the production of CXCL-10 in BMDCs, we treated the cells with various concentrations of FC-98 for $2 \mathrm{~h}$. After stimulation with $\mathrm{CpG}$ for additional $6 \mathrm{~h}$, we extracted the total RNA and examined the expression of CXCL-10 on transcription level. As shown in Figure 4(a), FC-98 could markedly decrease the mRNA expression of CXCL-10 in DCs at different dosages. Moreover, we treated cells with $\mathrm{CpG}$ for $24 \mathrm{~h}$ and the culture supernatants were collected and analyzed by ELISA. The similar results were found in the protein level of CXCL-10 (Figure 4(b)).

Since CXCL-10 plays an important role in the migration of T cell [21], we tried to clarify the effect of FC-98 on the migration of $\mathrm{T}$ cells to BMDCs and chemotaxis assay was used. As shown in Figure 4(c), FC-98 alone had little effect on the migration of $\mathrm{T}$ cells compared to control group, while all three concentrations of FC-98 markedly inhibited CpGinduced migration of T cells to BMDCs.

3.4. FC-98 Inhibited CXCL-10 through MAPK and STAT1 Signaling Pathway. Several studies reported that CXCL-10 expression is mediated through MAPK (ERK1/2, JNK, and p38) and STAT1 signaling pathway in several cell types [2224]. However, the regulation of FC-98 in BMDCs is unclear. So the mechanism underlying the inhibitory effect of FC98 on CXCL-10 was examined by detecting the activation of p38, JNK, ERK, and STAT1. Our previous articles had shown that the phosphorylation of p38, JNK, ERK, and STAT1 was sharply raised at $30 \mathrm{~min}$ when stimulated with $\mathrm{CpG}$ [16]. Thus BMDCs were treated with $100 \mu \mathrm{M}, 150 \mu \mathrm{M}$, or $200 \mu \mathrm{M}$ FC98 for $2 \mathrm{~h}$, followed by CpG stimulation at $1 \mu \mathrm{M}$ for $30 \mathrm{~min}$. Western blot data showed that FC-98 slightly inhibited the phosphorylation of JNK, p38, and ERK1/2 (Figure 5(a), left). To further confirm the inhibitory effects of FC-98 on MAPK signaling activation, BMDCs were transfected with an AP1-dependent luciferase gene reporter plasmid. As shown in Figure 5(a), right, pretreating cells with FC-98 displayed the reduced $\mathrm{CpG}$-induced luciferase activity, indicating a lower AP-1 activity. As to STAT1 signaling, FC-98 potently inhibited the phosphorylation of STAT1 (Figure 5(b)), whereas FC-98 had little influence on their total protein levels. These findings indicated that FC-98 inhibited the expression of CXCL-10 by reducing the activation of the MAPK and STAT1 signaling cascades in CpG-activated DCs.

\section{Discussion}

In the present study, we for the first time found that FC-98 regulated TLR9-mediated of CXCL-10 expression in BMDCss via MAPK and STAT1 signaling pathway. Using CCK8 assay and Annexin V/PI double staining, FC-98 below $200 \mu \mathrm{M}$ had no cytotoxicity on BMDCs. Upon CpG stimulation, FC-98 significantly downregulated the expression of CD40, CD80, CD86, and MHCII on the surface of BMDCs. In contrast, endocytosis of FITC-Dextran in the cells treated with FC-98 had no obvious difference between $4^{\circ} \mathrm{C}$ and $37^{\circ} \mathrm{C}$. Furthermore, FC-98 at different concentrations could markedly decrease the expression of CXCL-10 at both the mRNA and protein level in CpG-induced DCs. Meanwhile, FC-98 also suppressed the migration captivity related to 




(a)
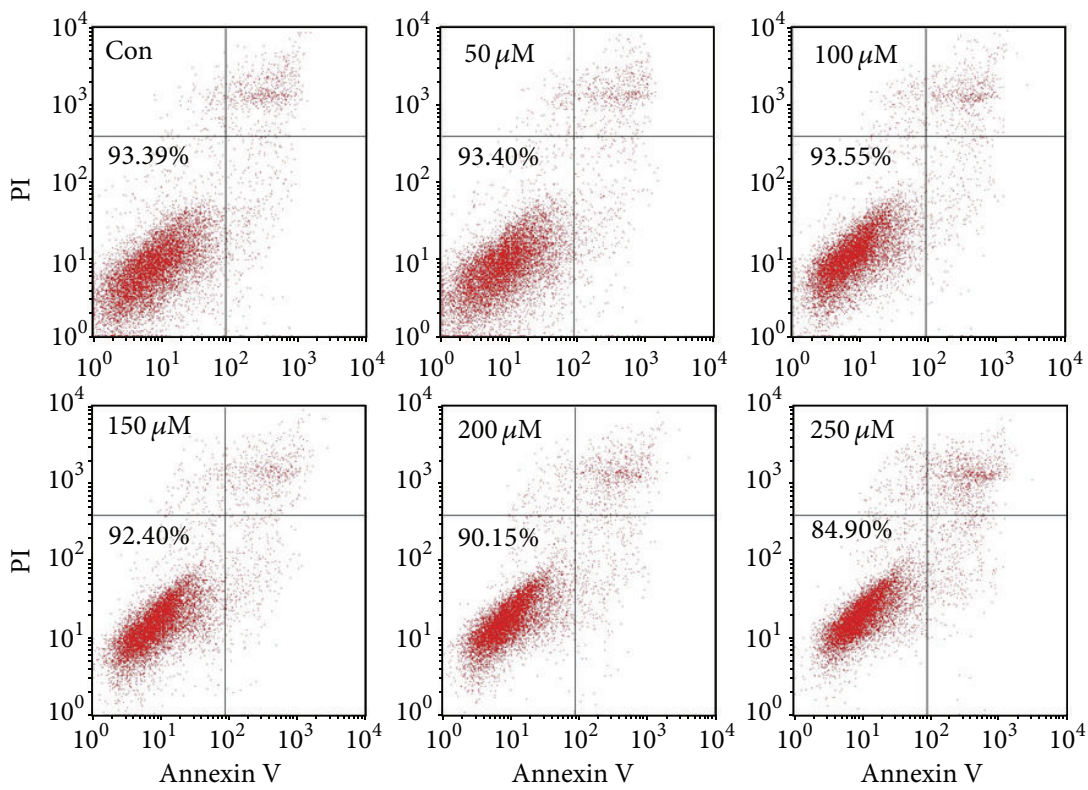

(b)

FIGURE 2: The effect of FC-98 on the cell viability and apoptosis of BMDCs. (a) BMDCs were treated with FC-98 in the range of 0-400 $\mu$ M for $24 \mathrm{~h}$ and then the CCK-8 assay was applied to detect the cell viability. (b) Cells were stained with Annexin V and PI followed by flow cytometry analyses. Cells stained with FITC-Annexin $\mathrm{V}$ alone $\left(\right.$ Annexin $\mathrm{V}^{+} / \mathrm{PI}^{-}$) were considered in early apoptosis, whereas those stained with both FITC-Annexin V and PI (Annexin $\mathrm{V}^{+} / \mathrm{PI}^{+}$) were considered in the advanced stages of apoptosis or necrosis. The FITC-Annexin V and PI double-negative (Annexin $\mathrm{V}^{-} / \mathrm{PI}^{-}$) cells were considered alive. Data are expressed as mean $\pm \mathrm{SD}$ of three independent experiments. ${ }^{* *} P<0.01$ versus control (Con) group.

CXCL-10 of T cells to BMDCs. Finally, we found that FC98 inhibited the activation of MAPK and STAT1 signaling pathway. All these data suggest that FC-98 may be a potential drug in the treatment of CXCL-10 mediated diseases.

DCs are the most potent professional antigen presenting cells and play a crucial role in promoting the natural immune and starting the adaptive immune [26]. DCs are derived from bone marrow progenitor cells and differentiate into immature DCs in lymphoid or nonlymphoid tissues [27]. When appropriate stimulations are identified, immature DCs can upregulate expression of cell surface markers (MHCII, CD40, CD80, and CD86) and proinflammatory cytokines and chemokines [28]. At the same time, their ability of uptaking Ag turns into presenting Ag [29]. Then mature
DCs activate T cells and regulate their responses [30]. DCs recognize pathogens by a set of pattern-recognition receptors. Among them, TLRs play an essential role [31]. CpG-ODN, which is similar to the bacterial DNA, can act as the TLR9 ligand to promote maturation and activation of DCs [32]. TLR9 are associated with various diseases such as autoimmune diseases and infectious diseases [33]. To date, TLRsbased therapeutics were more and more used in treatment of diseases [34].

Therefore, it is significant to regulate DCs maturation and function via the agents. Our present study had revealed several small molecule compounds to possess immunomodulatory properties on BMDCs via TLR signaling pathway $[16,35]$. Our laboratory had recently demonstrated the small 

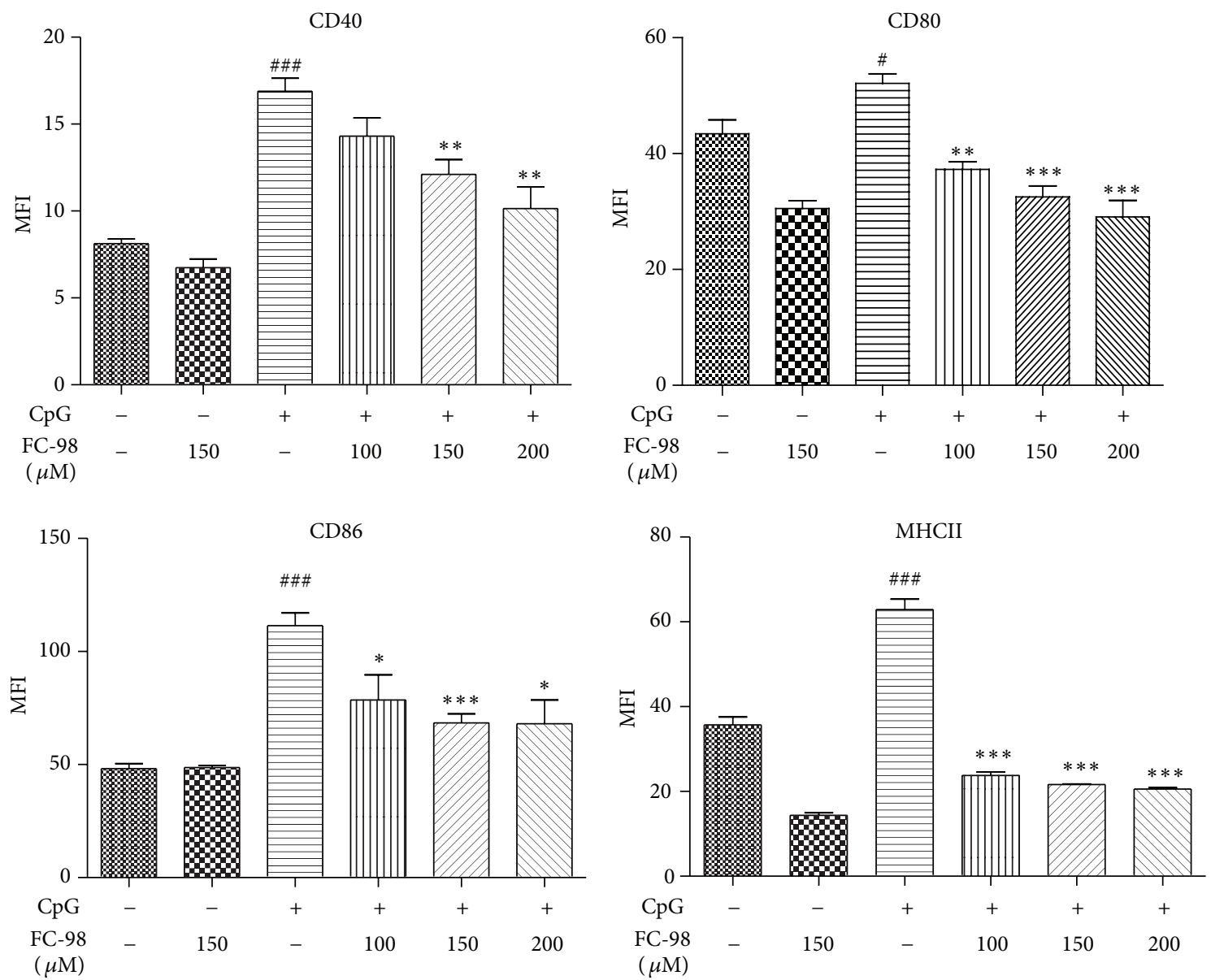

(a)
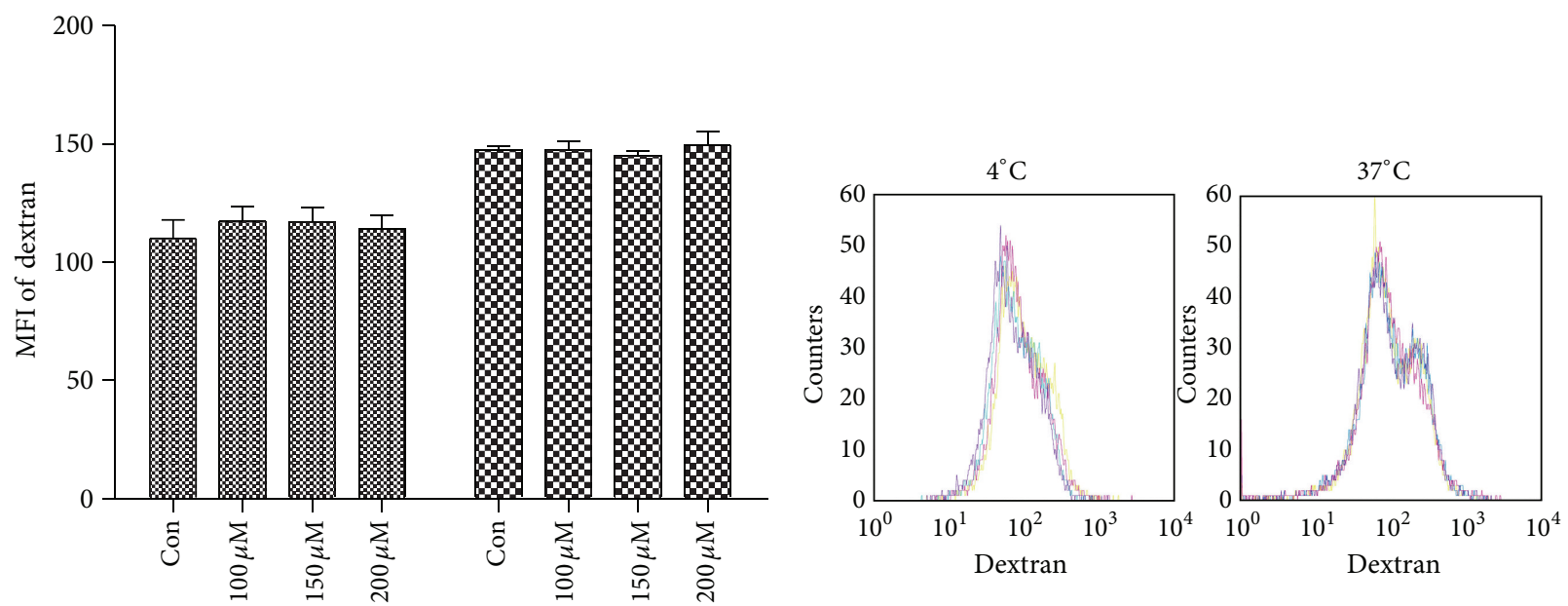

« $4^{\circ} \mathrm{C}$

$\operatorname{Bon} 37^{\circ} \mathrm{C}$

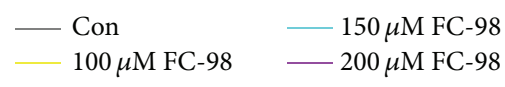

(b)

FIGURE 3: FC-98 inhibited CpG-induced upregulation of surface markers without influence on endocytosis. (a) BMDCs were treated with indicated concentrations of FC-98 for $2 \mathrm{~h}$, and then CpG $(1 \mu \mathrm{M})$ were added in. After $24 \mathrm{~h}$, the expressions of CD40, CD80, CD86, and MHCII were analyzed by flow cytometry. (b) BMDCs were treated with or without FC-98 and then $0.5 \mathrm{mg} / \mathrm{mL}$ FITC-Dextran was added for another $1 \mathrm{~h}$ at $37^{\circ} \mathrm{C}\left(4^{\circ} \mathrm{C}\right.$ as control $)$ and analyzed by flow cytometry. Data are shown as mean $\pm \mathrm{SD}$ of three independent experiments. ${ }^{\#} P<0.05$, ${ }^{\# \# \#} P<0.001$ versus control group; ${ }^{*} P<0.05,{ }^{* *} P<0.01$, and ${ }^{* * *} P<0.001$ versus $C p G$ only group. 


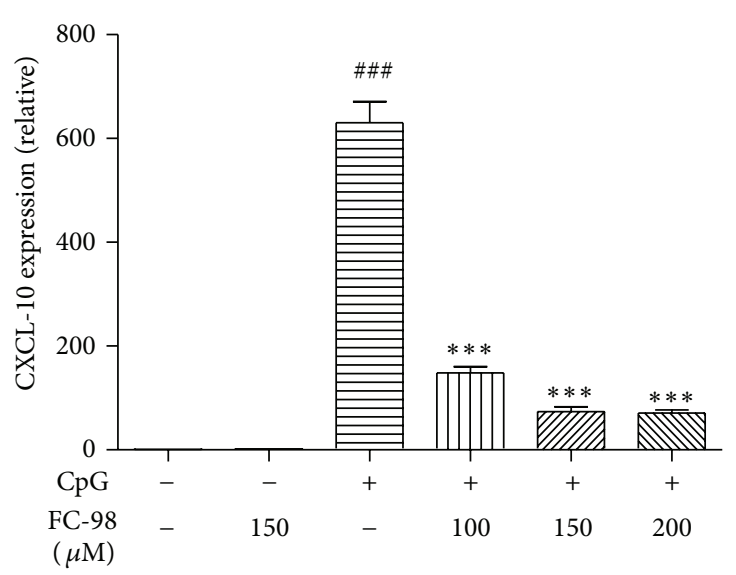

(a)



(b)

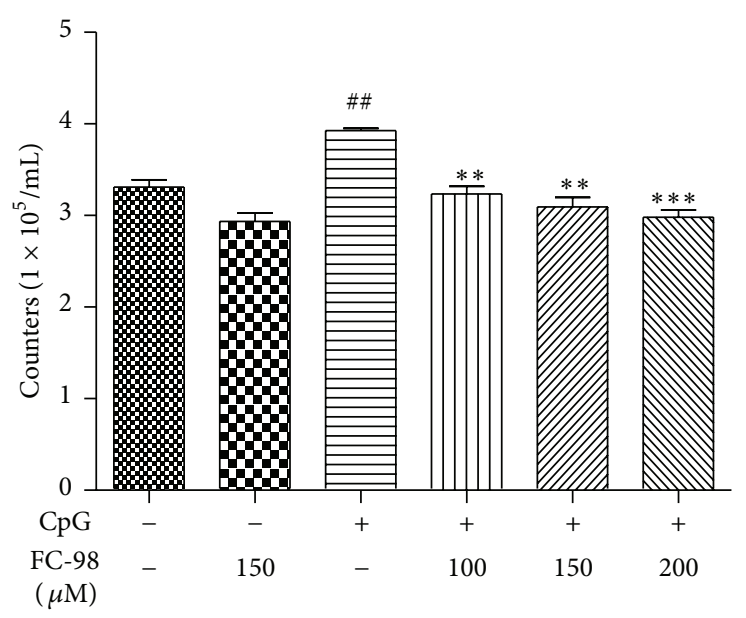

(c)

Figure 4: Effect of FC-98 on BMDCs CXCL-10 expression and function. BMDCs were treated with various concentrations of FC-98 for $2 \mathrm{~h}$, and then $1 \mu \mathrm{M} \mathrm{CpG}$ was added for another $6 \mathrm{~h}$ before q-PCR (a) or $24 \mathrm{~h}$ before ELISA (b). (c) Transwell experiment was performed to examine the function of pretreated BMDCs on T cells. The data are mean \pm SD of three independent experiments. ${ }^{\# \#} P<0.01$; ${ }^{\# \#} P<0.001$ a versus control group; ${ }^{* *} P<0.01 ;{ }^{* * *} P<0.001$ versus $\mathrm{CpG}$ only group.

molecule compound FC-98 which can inhibit the expression of TNF- $\alpha$, IL-6, MCP-1, and NO from LPS-induced mouse macrophages through attenuating the activation of NF- $\kappa \mathrm{B}$, JNK, and IRF3 signaling pathway. Moreover, FC-98 can effectively improve the survival rate and injured organ of mice's sepsis mode (unpublished). However, the immunoregulatory roles of FC-98 in the immune system remain needing further confirmation. The effect of FC-98 on the maturation and function of DCs remained unknown. In the present study, we revealed that FC-98 could significantly attenuate the phenotypic and functional maturation of $\mathrm{CpG}$-induced BMDCs. FC-98 could strongly inhibit the expression of MHC and costimulatory molecules (CD40, CD80, and CD86). Costimulatory molecules CD80/CD86 expressed on DCs can provide a co-stimulatory signal to T lymphocytes and induce expansion of antigen-specific $\mathrm{T}$ cells [36]. On the other hand, capability to uptake FITC-Daxtran of BMDCs was not affected by FC-98, suggesting that FC-98 might influence
BMDCs maturation via other ways not the endocytosis. These results suggested that FC-98 might be a potential inhibitor of DC maturation.

After maturation, DCs produce a serious of chemokines. Among them, CXCL-10 is critical to recruit and activate Th1polarized cells and has the abilities of antitumor, antiviral, and antifungal [37-40]. In DCs, CXCL-10 production in regulatory DCs can downregulate T-cell response and inhibition of recruited Thl cell proliferation [41]. CXCL-10 is also critical for rendering a protective cellular immunity during SLA-CpG-DC vaccination that confers protection against Leishmania donovani infection [42]. In our study, we found that BMDCs produced large amounts of CXCL-10 upon CpG stimulation and pretreatment of FC-98 could notably impair its upregulation at both mRNA and protein levels.

CXCL-10 is correlated with the tissue infiltration of T lymphocytes and mediates in the recruitment of $\mathrm{T}$ cells [43]. To further confirm the inhibitory effect of FC-98 on 



(a)
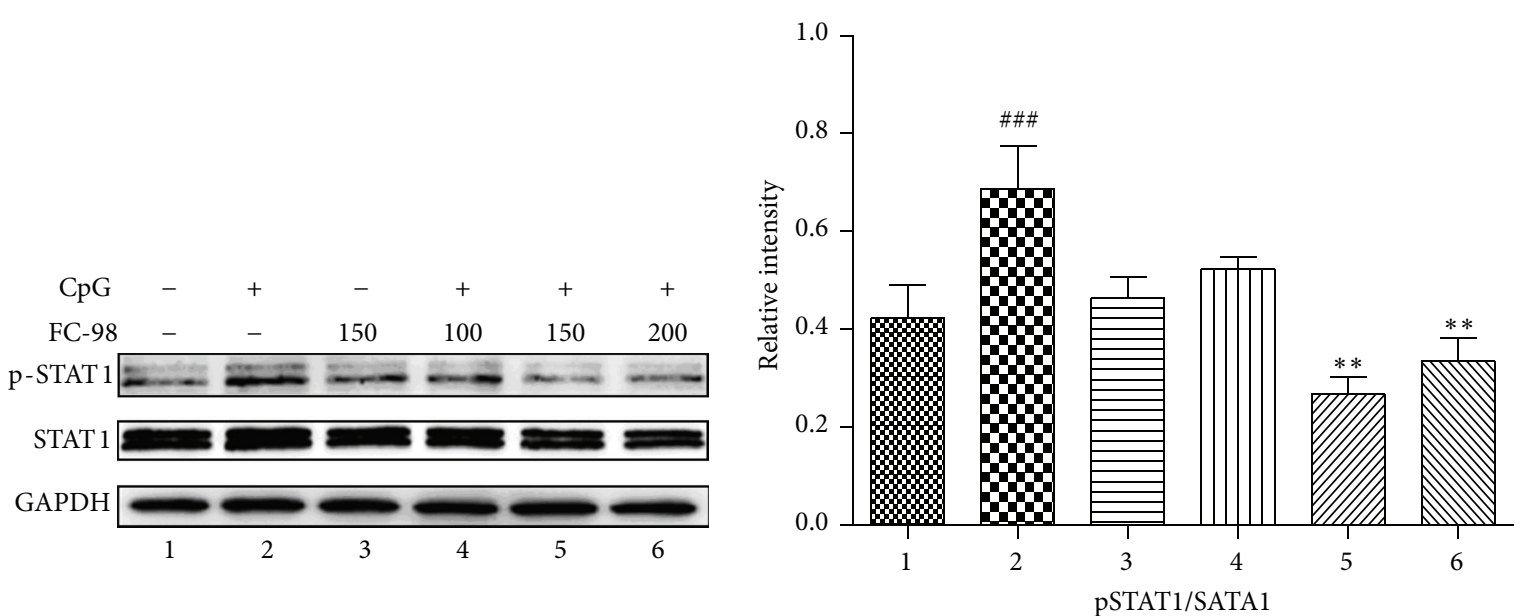

(b)

FIGURE 5: FC-98 inhibited activation of MAPK and STAT1 to downregulate the CXCL-10 expression. (a) Left: BMDCs were pretreated with FC-98 for $2 \mathrm{~h}$, followed by 30 min CpG treatment; the phosphorylation of of MAPK (ERK, JNK, and p38) signaling pathway was analyzed by western blot. The results shown are representative experiments from three independent assays. Right: BMDCs were cotransfected with $100 \mathrm{ng}$ pAP-1 luciferase reporter plasmid and $10 \mathrm{ng}$ pRL-TK-Renilla luciferase. Total amounts of plasmid DNA were equalized using empty control vector. After $24 \mathrm{~h}$ of culture, cells were pretreated with FC-98 for $1 \mathrm{~h}$ and then stimulated with $1 \mu \mathrm{M}$ CpG for another $6 \mathrm{~h}$. Luciferase activity was measured and normalized by Renilla luciferase activity. Data are shown as mean \pm SD of three independent assays. ${ }^{\# \# \#} P<0.001$ versus control group; ${ }^{*} P<0.05,{ }^{* * *} P<0.001$ versus CpG-only group. (b) Left: experiments were duplicated as described in (a), left, and phosphorylated STAT1 (p-STAT1) and total STAT1 were detected by western blot. The results shown are representative experiments from three independent assays. Right: the results of western blot were analysed by Image J, and the relative intensity was showed. ${ }^{\# \#} P<0.001$ versus control group; $* * P<0.01$ versus 2 (CpG-only group).

CXCL-10, we also examined the effect of the BMDCs culture supernatant on the $\mathrm{T}$ cells migration. The results showed that FC-98 predominantly inhibited the T cell migration induced by CpG. Thus, it is possible that FC-98 might inhibit the chemokine CXCL-10 expression of DCs, further inhibiting the recruitment of T cells.

As to the mechanisms of FC-98 downregulating CXCL10 production, we explored the MAPK and STAT1 signaling pathways. Williams R found that the upregulation of CXCL10 at both mRNA and protein levels is mediated by the activation of the p38, JNK signaling pathways, and along with the activation of STAT1 [44]. In murine macrophage-like cells, the upregulation of CXCL10 is associated with the activation of JAK1, JAK2/STAT1, and ERK1/2 [22, 24]. In cancer researches, expression of CXCL10 is mediated through the Raf, PI3K, p38/MAPK, JNK/MAPK, and NF- $\kappa$ B signaling cascades [45]. Taken together, we are interested in the MAPK and STAT1 signaling pathway. We pretreated BMDCs with FC-98 for $2 \mathrm{~h}$ and then CpG were added for another $30 \mathrm{~min}$ and the cell lysis was collected. Western blot experiment revealed that FC-98 
inhibited the phosphorylation of JNK, ERK, p38, and STAT1. Luciferase report assay of AP-1 activation was also applied to confirm the results. Collectively, these findings suggest that FC-98 inhibits the expression of CXCL-10 in CpG-induced BMDCs via the MAPK (JNK, ERK, and p38) and STAT1 signaling pathways.

In conclusion, our present study characterized a small molecule FC-98 regulating BMDCs maturation via CXCL-10. Thus, FC-98 may be potential in the treatment of CXCL-10associated diseases.

\section{Conflict of Interests}

The authors declare that there is no conflict of interests regarding the publication of this paper.

\section{Acknowledgments}

This work was supported by the National Natural Science Foundation of China (no. 31370899, no. 81121062, and no. 31128006). Wei Gong was supported by the Scientific Research Foundation of Graduate School of Nanjing University (2012CL03).

\section{References}

[1] R. M. Steinman and H. Hemmi, "Dendritic cells: translating innate to adaptive immunity," Current Topics in Microbiology and Immunology, vol. 311, pp. 17-58, 2006.

[2] Y. Hiasa, S. M. F. Akbar, M. Abe, K. Michitaka, N. Horiike, and M. Onji, "Dendritic cell subtypes in autoimmune liver diseases; decreased expression of HLA DR and CD123 on type 2 dendritic cells," Hepatology Research, vol. 22, no. 4, pp. 241-249, 2002.

[3] B. J. O'Sullivan, S. Pai, S. Street et al., "Immunotherapy with costimulatory dendritic cells to control autoimmune inflammation," Journal of Immunology, vol. 187, no. 8, pp. 4018-4030, 2011.

[4] J. E. Boudreau, A. Bonehill, K. Thielemans, and Y. Wan, "Engineering dendritic cells to enhance cancer immunotherapy," Molecular Therapy, vol. 19, no. 5, pp. 841-853, 2011.

[5] F. Angelot, E. Seillès, S. Biichlé et al., "Endothelial cell-derived microparticles induce plasmacytoid dendritic cell maturation: potential implications in inflammatory diseases," Haematologica, vol. 94, no. 11, pp. 1502-1512, 2009.

[6] F. Xing, J. Wang, M. Hu, Y. Yu, G. Chen, and J. Liu, “Comparison of immature and mature bone marrow-derived dendritic cells by atomic force microscopy," Nanoscale Research Letters, vol. 6, article 455, pp. 1-9, 2011.

[7] C. Reis e Sousa, "Dendritic cells in a mature age," Nature Reviews Immunology, vol. 6, no. 6, pp. 476-483, 2006.

[8] N. S. Wilson, G. M. N. Behrens, R. J. Lundie et al., "Systemic activation of dendritic cells by Toll-like receptor ligands or malaria infection impairs cross-presentation and antiviral immunity," Nature Immunology, vol. 7, no. 2, pp. 165-172, 2006.

[9] A. Iwasaki and R. Medzhitov, "Toll-like receptor control of the adaptive immune responses," Nature Immunology, vol. 5, no. 10, pp. 987-995, 2004.

[10] L. A. O’Neill, D. Golenbock, and A. G. Bowie, “The history of Toll-like receptors-redefining innate immunity," Nature Reviews Immunology, vol. 13, no. 6, pp. 453-460, 2013.
[11] C. Reis e Sousa, "Toll-like receptors and dendritic cells: for whom the bug tolls," Seminars in Immunology, vol. 16, no. 1, pp. 27-34, 2004.

[12] T. Tokunaga, H. Yamamoto, and S. Shimada, "Antitumor activity of deoxyribonucleic acid fraction from Mycobacterium bovis BCG. I. Isolation, physicochemical characterization, and antitumor activity," Journal of the National Cancer Institute, vol. 72, no. 4, pp. 955-962, 1984.

[13] C. Coban, K. J. Ishii, T. Kawai et al., “Toll-like receptor 9 mediates innate immune activation by the malaria pigment hemozoin," Journal of Experimental Medicine, vol. 201, no. 1, pp. 19-25, 2005.

[14] A. Trieu, T. L. Roberts, J. A. Dunn, M. J. Sweet, and K. J. Stacey, "DNA motifs suppressing TLR9 responses," Critical Reviews in Immunology, vol. 26, no. 6, pp. 527-544, 2006.

[15] R. J. Dearman, M. Cumberbatch, G. Maxwell, D. A. Basketter, and I. Kimber, "Toll-like receptor ligand activation of murine bone marrow-derived dendritic cells," Immunology, vol. 126, no. 4, pp. 475-484, 2009.

[16] C. Hua, Y. Yang, L. Sun, H. Dou, R. Tan, and Y. Hou, "Chaetoglobosin F, a small molecule compound, possesses immunomodulatory properties on bone marrow-derived dendritic cells via TLR9 signaling pathway," Immunobiology, vol. 218, no. 3, pp. 292-302, 2013.

[17] A. O. Weinzierl, G. Szalay, H. Wolburg et al., "Effective chemokine secretion by dendritic cells and expansion of crosspresenting $\mathrm{CD}^{-} / \mathrm{CD}^{+}$dendritic cells define a protective phenotype in the mouse model of coxsackievirus myocarditis," Journal of Virology, vol. 82, no. 16, pp. 8149-8160, 2008.

[18] E. Y. Lee, Z.-H. Lee, and Y. W. Song, "CXCL10 and autoimmune diseases," Autoimmunity Reviews, vol. 8, no. 5, pp. 379-383, 2009.

[19] M. Liu, S. Guo, J. M. Hibbert et al., "CXCL10/IP-10 in infectious diseases pathogenesis and potential therapeutic implications," Cytokine and Growth Factor Reviews, vol. 22, no. 3, pp. 121-130, 2011.

[20] W. A. Dar and S. J. Knechtle, "CXCR3-mediated T-cell chemotaxis involves ZAP-70 and is regulated by signalling through the T-cell receptor," Immunology, vol. 120, no. 4, pp. 467-485, 2007.

[21] U. Pertl, A. D. Luster, N. M. Varki et al., "IFN- $\gamma$-inducible protein-10 is essential for the generation of a protective tumorspecific CD8 T cell response induced by single-chain IL-12 gene therapy," Journal of Immunology, vol. 166, no. 11, pp. 6944-6951, 2001.

[22] K. Nakamichi, S. Inoue, T. Takasaki, K. Morimoto, and I. Kurane, "Rabies virus stimulates nitric oxide production and CXC chemokine ligand 10 expression in macrophages through activation of extracellular signal-regulated kinases 1 and 2," Journal of Virology, vol. 78, no. 17, pp. 9376-9388, 2004.

[23] A. Bonacchi, P. Romagnani, R. G. Romanelli et al., "Signal transduction by the chemokine receptor CXCR3: activation of Ras/ERK, Src, and phosphatidylinositol 3-kinase/Akt controls cell migration and proliferation in human vascular pericytes," The Journal of Biological Chemistry, vol. 276, no. 13, pp. 99459954, 2001.

[24] C. Han, J. Fu, Z. Liu, H. Huang, L. Luo, and Z. Yin, "Dipyrithione inhibits IFN- $\gamma$-induced JAK/STAT1 signaling pathway activation and IP-10/CXCL10 expression in RAW264.7 cells," Inflammation Research, vol. 59, no. 10, pp. 809-816, 2010.

[25] R. M. Kyung, H.-M. Shin, J.-H. Lee et al., "Anti-inflammatory effects of N1-benzyl-4-methylbenzene-1,2- diamine (JSH-21) 
analogs on nitric oxide production and nuclear factor-kappa B transcriptional activity in lipopolysaccharide-stimulated macrophages RAW 264.7," Archives of Pharmacal Research, vol. 27, no. 10, pp. 1053-1059, 2004.

[26] M. Merad and M. G. Manz, "Dendritic cell homeostasis," Blood, vol. 113, no. 15, pp. 3418-3427, 2009.

[27] J. Banchereau and R. M. Steinman, "Dendritic cells and the control of immunity, Nature, vol. 392, no. 6673, pp. 245-252, 1998.

[28] S. Akira, S. Uematsu, and O. Takeuchi, "Pathogen recognition and innate immunity," Cell, vol. 124, no. 4, pp. 783-801, 2006.

[29] S. J. van Vliet, J. D. Dunnen, S. I. Gringhuis, T. B. Geijtenbeek, and Y. van Kooyk, "Innate signaling and regulation of Dendritic cell immunity," Current Opinion in Immunology, vol. 19, no. 4, pp. 435-440, 2007.

[30] B. J. C. Quah and H. C. O'Neill, "Maturation of function in dendritic cells for tolerance and immunity," Journal of Cellular and Molecular Medicine, vol. 9, no. 3, pp. 643-654, 2005.

[31] S. Akira, K. Takeda, and T. Kaisho, "Toll-like receptors: critical proteins linking innate and acquired immunity," Nature Immunology, vol. 2, no. 8, pp. 675-680, 2001.

[32] F. H. A. Fakher, N. Rachinel, M. Klimczak, J. Louis, and N. Doyen, "TLR9-dependent activation of dendritic cells by DNA from Leishmania major favors TH1 cell development and the resolution of lesions," Journal of Immunology, vol. 182, no. 3, pp. 1386-1396, 2009.

[33] J. Vollmer and A. M. Krieg, "Immunotherapeutic applications of CpG oligodeoxynucleotide TLR9 agonists," Advanced Drug Delivery Reviews, vol. 61, no. 3, pp. 195-204, 2009.

[34] A. Dunne, N. A. Marshall, and K. H. G. Mills, "TLR based therapeutics," Current Opinion in Pharmacology, vol. 11, no. 4, pp. 404-411, 2011.

[35] L. Sun, C. Hua, Y. Yang et al., "Chaeoglobosin Fex inhibits poly(I:C)-induced activation of bone marrow-derived dendritic cells," Molecular Immunology, vol. 51, no. 2, pp. 150-158, 2012.

[36] M. B. Lutz and G. Schuler, "Immature, semi-mature and fully mature dendritic cells: which signals induce tolerance or immunity?" Trends in Immunology, vol. 23, no. 9, pp. 445-449, 2002.

[37] L. E. Rosas, J. Barbi, B. Lu et al., "CXCR3-/- mice mount an efficient Th1 response but fail to control Leishmania major infection," European Journal of Immunology, vol. 35, no. 2, pp. 515-523, 2005.

[38] S. V. Maru, K. A. Holloway, G. Flynn et al., "Chemokine production and chemokine receptor expression by human glioma cells: role of CXCL10 in tumour cell proliferation," Journal of Neuroimmunology, vol. 199, no. 1-2, pp. 35-45, 2008.

[39] A. Azzurri, O. Y. Sow, A. Amedei et al., "IFN- $\gamma$-inducible protein 10 and pentraxin 3 plasma levels are tools for monitoring inflammation and disease activity in Mycobacterium tuberculosis infection," Microbes and Infection, vol. 7, no. 1, pp. 1-8, 2005.

[40] H. Kabashima, M. Yoneda, K. Nagata, T. Hirofuji, and K. Maeda, "The presence of chemokine (MCP-1, MIP-1 $\alpha$, MIP-1 $\beta$, IP10, RANTES)-positive cells and chemokine receptor (CCR5, CXCR3)-positive cells in inflamed human gingival tissues," Cytokine, vol. 20, no. 2, pp. 70-77, 2002.

[41] C. Qian, H. An, Y. Yu, S. Liu, and X. Cao, “TLR agonists induce regulatory dendritic cells to recruit Th1 cells via preferential IP10 secretion and inhibit Th1 proliferation," Blood, vol. 109, no. 8, pp. 3308-3315, 2007.
[42] S. Majumder, S. Bhattacharjee, C. B. Paul, and S. Majumdar, "CXCL10 is critical for the generation of protective CD8 T cell response induced by antigen pulsed CpG-ODN activated dendritic cells," PLoS ONE, vol. 7, no. 11, 2012.

[43] U. Christen, D. Benke, T. Wolfe et al., "Cure of prediabetic mice by viral infections involves lymphocyte recruitment along an IP10 gradient," The Journal of Clinical Investigation, vol. 113, no. 1, pp. 74-84, 2004.

[44] R. Williams, H. Yao, N. K. Dhillon, and S. J. Buch, "HIV-1 Tat cooperates with IFN- $\gamma$ and TNF- $\alpha$ to increase CXCL10 in human astrocytes," PLoS ONE, vol. 4, no. 5, Article ID e5709, 2009.

[45] Q. Shen, R. Zhang, and N. R. Bhat, "MAP kinase regulation of IP10/CXCL10 chemokine gene expression in microglial cells," Brain Research, vol. 1086, no. 1, pp. 9-16, 2006. 


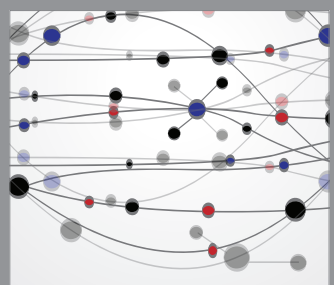

The Scientific World Journal
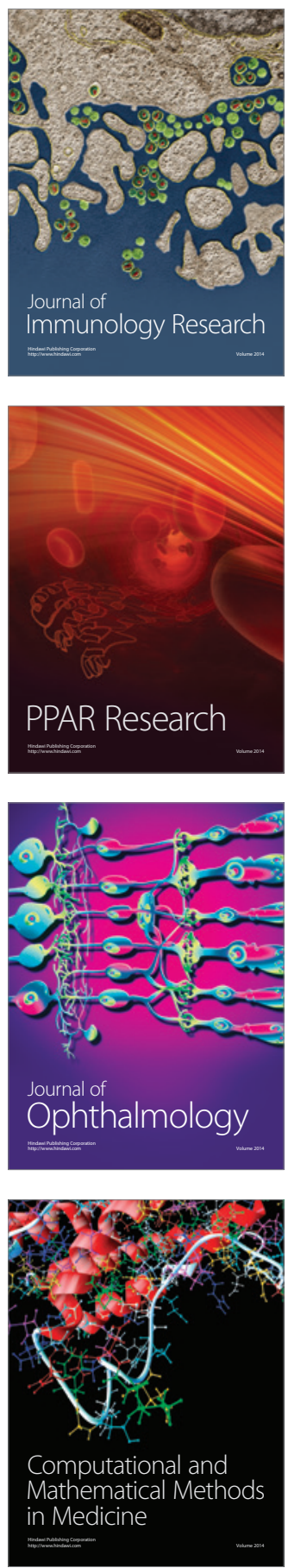

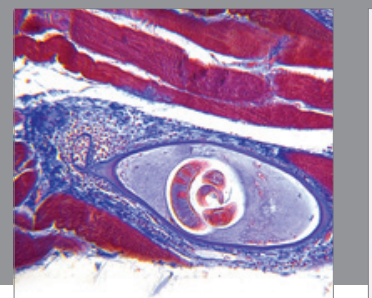

Gastroenterology

Research and Practice
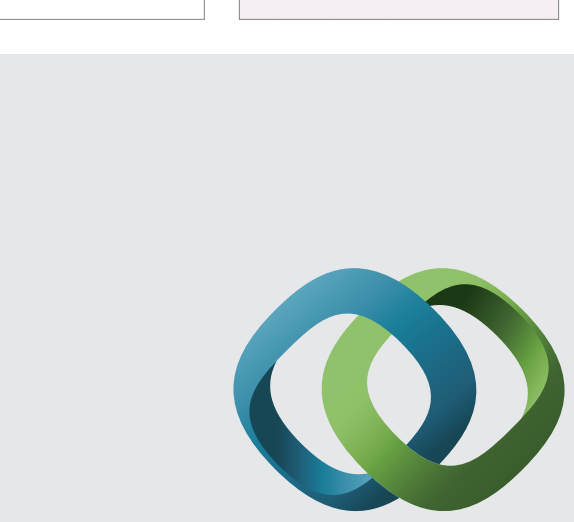

\section{Hindawi}

Submit your manuscripts at

http://www.hindawi.com
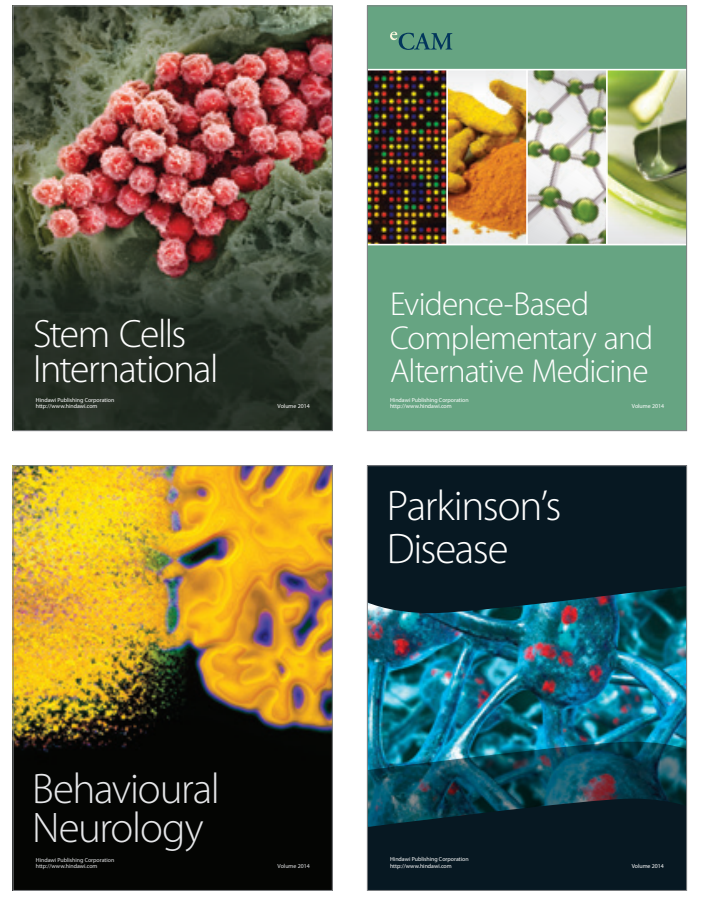
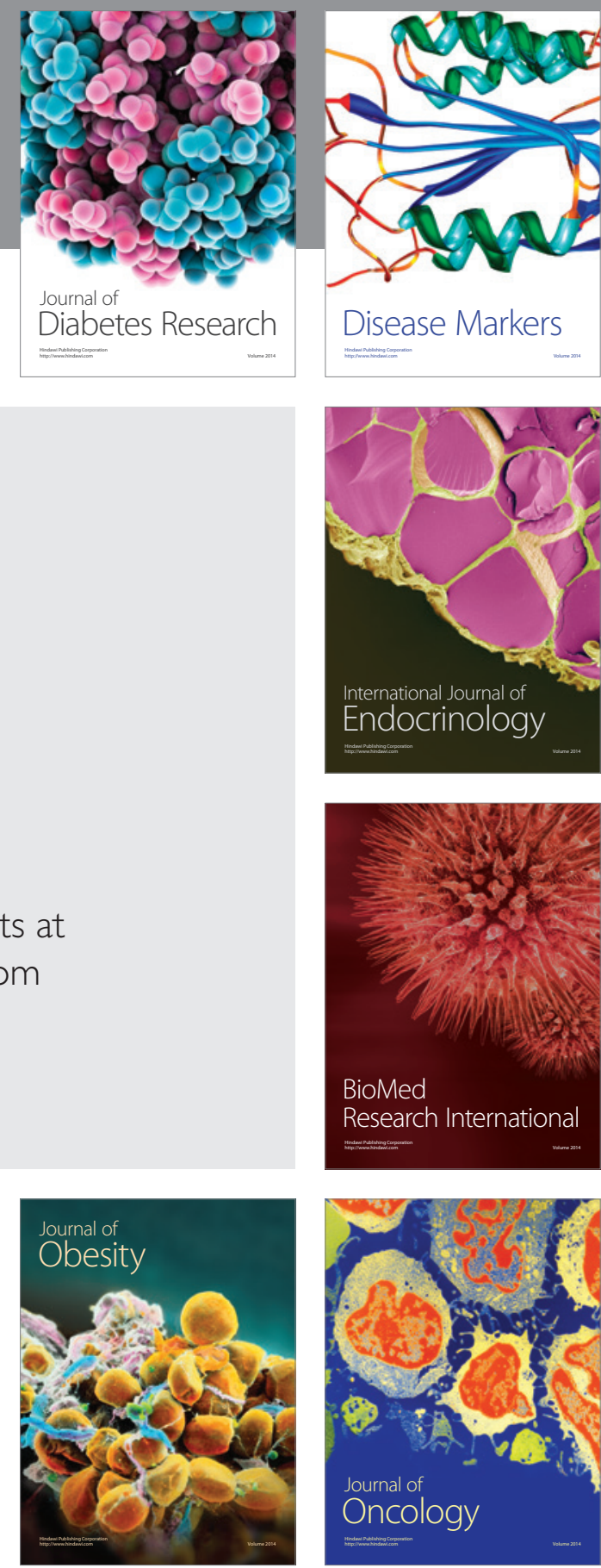

Disease Markers
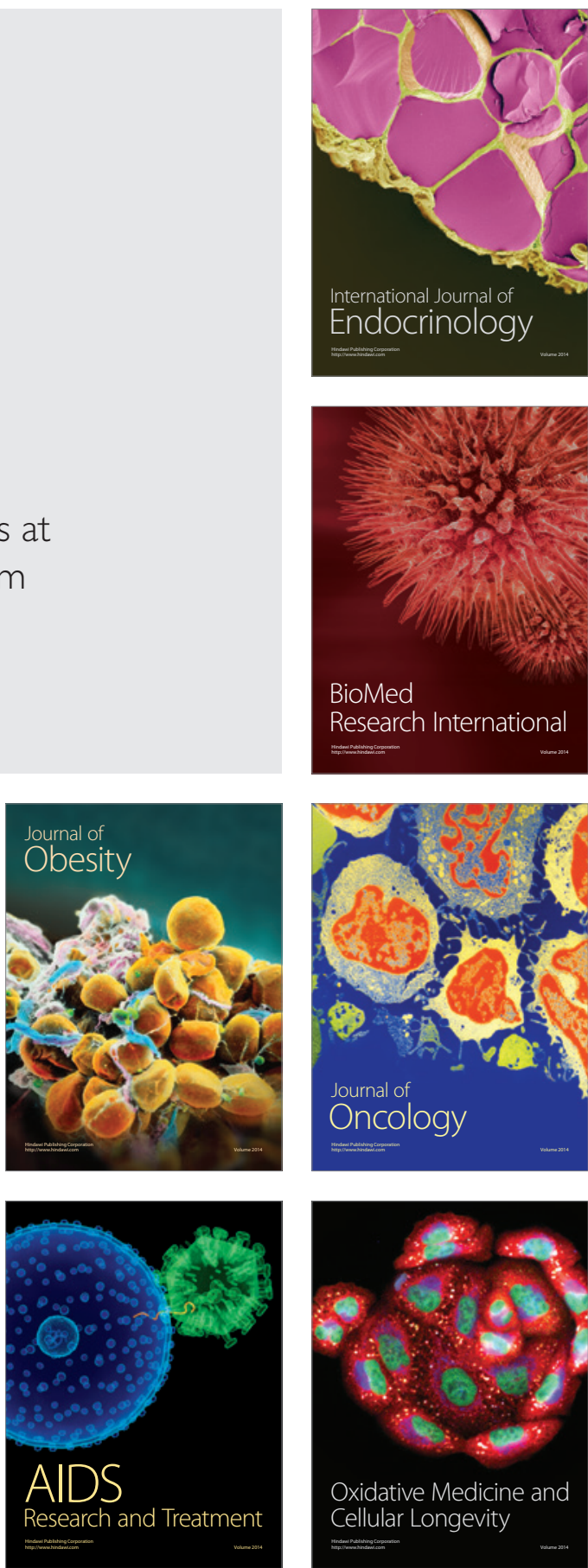